\title{
The Power of Partnerships: State Public Health Department Multisector Collaborations in Major Chronic Disease Program Areas
}

Edward Tsai ( $\boldsymbol{\sigma}$ etsaia@wustl.edu )

Washington University School of Medicine in St. Louis https://orcid.org/0000-0002-5363-9145

Peg Allen

Washington University in St Louis

Louise F Saliba

Washington University in St Louis

Ross C Brownson

Washington University in St Louis

\section{Research}

Keywords: public health, state health department, chronic disease, multisector collaboration, health equity

Posted Date: March 12th, 2021

DOl: https://doi.org/10.21203/rs.3.rs-282598/v1

License: (c) (i) This work is licensed under a Creative Commons Attribution 4.0 International License.

Read Full License 


\section{Abstract \\ Background}

Multisector collaboration between state health departments (SHDs) and diverse community partners is increasingly recognized as important for promoting positive public health outcomes, addressing social determinants of health, and reducing health inequalities. This study investigates collaborations between SHDs and different types of organizations addressing chronic disease in and outside of the health sector.

\section{Methods}

SHD employees were randomly selected from the National Association of Chronic Disease Directors membership list for participation in an online survey. Participants were asked about their primary chronic disease work unit (cancer, obesity, tobacco, diabetes, cardiovascular disease, and others), as well as their work unit collaborations (exchange of information/cooperation in activities) with organizations in health and non-health sectors. As a measure of the different organizations SHDs collaborated with in health and non-health sectors, a collaboration heterogeneity score for each program area was calculated. One-way ANOVA with Tukey's post hoc tests were used to assess differences in collaborator heterogeneity between program areas.

\section{Results}

A total of 574 participants were surveyed. Results indicated the cancer program area, along with diabetes and cardiovascular disease, had significantly less collaboration heterogeneity with organizations outside of the health sector compared to the obesity and tobacco program areas.

\section{Conclusions}

Evidence suggests that to address social determinants of health and promote health equity, increased public health collaborations with diverse non-health sector community-based settings is critical.

\section{Background}

Multisector interagency collaboration is necessary to implement the complex multi-level evidence-based health policies, environmental interventions, and systems approaches that address social determinants of health for the major chronic diseases that have become a core focus for public health $[1,2]$. Governmental public health departments have increasingly collaborated with other health organizations such as clinics and hospitals, with calls for increased partnerships with a broader array of organizations [3]. In recent decades, the Department of Health and Human Services (DHHS) through the Healthy People objectives, along with the Institute of Medicine (IOM, now the National Academy of Medicine), have 
continuously advocated for stronger collaborations between public health departments and multisector organizations. For example, core Healthy People agenda items starting from the turn of the century have called for state health departments (SHDs) to take a leadership role in collaborating with diverse partners to facilitate the implementation of community health improvement plans [4]. In the same timeframe, IOM recommendations from annual reports have called for collaborative partnerships as a key mechanism for involving organizations with a stake in community health [5].

Despite this, the extent of widespread public health department collaboration with multisector organizations remains unclear, with some evidence suggesting multisector partnerships are beneficial for community health, but that public health departments still collaborate primarily with organizations within the health sector $[4,6,7]$. Participants in Canada's Multi-Sector Partnerships Initiative in public health reported increased resources, including increased access to people with different skills and expertise [8, 9]. Additionally, most studies in the area of public health collaborations have focused on primarily characterizing smaller-scale partnerships between local level health departments and agencies [5]. Less is known relatively on how mid to higher level public health agencies such as SHDs collaborate with organizations outside of the health sector. Evidence at the local level shows benefits of multisector community partnerships. For example, a national study by Tabak et al. (2018) found that for local health departments, collaboration with other multisector organizations in the community was critical for the provision of evidence-based interventions related to obesity and diabetes prevention, as few interventions were delivered only directly by the local health department itself [10]. In the area of cardiovascular disease, a local multisector initiative resulted in increased percentages of healthcare system hypertensive patients with controlled blood pressure, compared to baseline [11]. Multisector cancer collaborations have shown increased use of evidence-based approaches to facilitate cancer screening $[12,13]$ and increased cancer screening rates [14]. While hospitals are required to work with community-based organizations to inform their community health needs assessments and plans, such collaborations are often not sustained through implementation [15].

While evidence in local-level partnerships is promising, key differences exist in the roles and collaborative and partnership forming processes of state-level health departments versus local level health agencies. For example, compared to local health departments, SHDs are expected to take on a more central leadership role, to be involved in higher-level activities such as informing state-wide health policy creation, and to manage relationships with diverse partner organizations and many different local health departments that may have competing priorities $[5,16]$.

Additionally, SHDs increasingly focus on health equity at a systems level and can serve as a bridging hub to foster both state and local level multisector collaborations to address health equity and social determinants of health. Because social determinants of health are impacted by a diverse array of service sectors outside of health-focused organizations like hospitals and clinics (e.g. housing, transportation, schools, city planning), SHDs are often in a position to facilitate partnerships between organizations belonging to different sectors [3]. In the context of community health equity, evidence suggests that in state-level public health practice, a positive association exists between higher quality, more diverse 
partnerships and commitment to health equity work [17]. A recent systematic review on public health strategies to reduce health inequalities additionally identified multisector collaborations by public health agencies as a core component for successful interventions and programs [18].

The Association of State and Territorial Health Officials (ASTHO) report on multisector collaboration among state health agencies provides the best picture of U.S. state-level health agency collaboration in the literature [6]. This is a valuable and critical source of information on health agency collaborations at the state level, but the ASTHO survey assesses collaboration with a broad lens and does not break down multisector collaborations by chronic disease program area or work unit within each SHD. This is a gap, as SHDs are not monolithic entities and may contain numerous program area work units focused on different infectious and chronic diseases. Depending on the specific chronic disease area, these different work units will be engaged in different types of health-promoting activities, thereby necessitating different types of organizational partners and collaborators. For example, a public health work unit focused on reducing childhood obesity may be more likely to collaborate with the parks and recreation department (to promote outdoor physical activity) compared to a work unit in the tobacco control program area.

This paper aims to provide a snapshot of the types of organizations SHDs collaborate with for chronic disease prevention, the degree to which collaborating organizations lie in the health sector versus other sectors, and if/how collaborations differ depending on specific chronic disease program areas. Therefore, this study adds to the growing body of literature on multisector collaboration in that most existing research has focused on local level health department partnerships, studies that are on a state level are primarily focused on the SHD collaborations within a single state, and studies on SHDs nationally have yet to characterize collaborations within specific chronic disease program areas.

\section{Methods}

The survey used was part of a larger study investigating factors associated with mis-implementation of public health programs in SHDs. The study protocol for this larger overall project has been previously reported [19]. Participants for the survey were SHD employees recruited from the National Association of Chronic Disease Directors (NACDD) membership list working in SHD chronic disease units. Participants were randomly selected from the NACDD membership list after individuals from territories and nonqualifying positions (e.g., clerical and financial personnel) were excluded. Emails were sent out in June 2018 inviting a random sample of 1239 members (of 3117) to participate in a Qualtrics online survey that remained open for participation until August 2018. Participants were offered a \$20 Amazon gift card or to have a donation made to a public health charity of their choosing. Human subjects approval was obtained from the Washington University in St. Louis Institutional Review Board (\#201812062).

\section{Measures}

The survey included items on respondent demographics, chronic disease program working area, experience, and training. The final survey draft underwent cognitive response testing with 11 former SHD 
chronic disease directors. Reliability test-retest of the revised draft with 39 current SHD chronic disease unit staff found consistency in scores, and only minor changes to the survey were needed.

Type of organizations SHDs collaborated with was assessed by providing a roster of possible organization types with the prompt "Which types of organizations does your work unit currently collaborate with?" For each potential partner organization type, respondents were given the option to select any of "exchange information," "work together on activities or projects," "my agency provides financial resources," "my agency serves in a leadership role in the collaboration," and "my agency is a recipient of financial resources from this organization" to indicate the specific nature of collaboration. Two sets of rosters were provided; one with a list of potential collaborating organizations in the health sector (local health departments, other SHDs, clinics/FQHCs, hospitals, universities/schools/departments focused on health, Indian Health Service, tribal health organizations, Medicaid unit of state agency, state medical associations, health non-profits, health insurance providers, mental health services, foundation/public health institutes), and another with potential collaborators in non-health sectors (universities/schools/departments non-health focused, pre k-12 education/youth programs, media/communications/public relation organizations, community development organizations, social services other than Medicaid, businesses, parks, and recreation departments, housing, city planning/transportation agencies, justice system, state commissions/special counsels, tribal agencies, other state agencies).

\section{Analysis}

Analysis was conducted on the following chronic disease program areas: cancer prevention and control, obesity, tobacco, diabetes, and cardiovascular disease. A sixth category was included titled "multiple program areas," which was the option respondents selected if they worked in more than one program area, as many SHD program managers and section leaders do. Based on the two survey rosters provided, all possible collaborating organization types were categorized into one of two sectors, either "health sector" (including organizations providing healthcare-related services) or "multisector" (including organizations not directly providing health services or not in healthcare-related fields). Collaboration was defined as if a respondent indicated they exchanged information and/or worked together on activities or projects with a particular organization.

Collaboration survey responses were combined for each SHD chronic disease program area so that in cases where there was more than one respondent for any particular program area in a given state, their responses for organizations collaborated with were aggregated into a single case for that state. Table 1 contains the initial total collected sample size for each chronic disease program area, as well as the aggregated sample size used for analysis in Figs. 1-6, and all appendix figures. As a measure for heterogeneity of organizations collaborated with in the health sector versus multisector, the probability that any two randomly selected organizations collaborating with a SHD in a program area would be in different sectors was calculated, normalized to a 0-1 scale. Therefore, the higher this heterogeneity number (i.e., the closer the value to 1 ), the greater the likelihood that any two randomly selected collaborators for a SHD program area would be from different sectors, indicating greater heterogeneity 
(i.e. multisector collaborations) in the types of organizations that SHD program area is working with. This measure, known as the Agresti index of qualitative variation, is a commonly used statistic in fields including sociology, biology, and public health to indicate heterogeneity/diversity with respect to any particular characteristic among a group of entities, for example, gender heterogeneity among a group of students, species diversity in a habitat, and in this case, the heterogeneity of organizational sector types SHDs collaborate with $[20,21]$. ANOVA with Tukey's HSD post hoc test was conducted to assess potential significant differences in heterogeneity of collaborators across the six chronic disease program areas. Analysis and figures were conducted using SPSS 26 and E-Net [22].

Table 1

Inter-organizational collaboration scores by program area in a 2018 survey of state health department health promotion/chronic disease units, United States.

\begin{tabular}{|lllc|}
\hline Program Area & $\begin{array}{l}\text { Total } \\
\text { number of } \\
\text { respondents }\end{array}$ & $\mathbf{N}^{1}$ & $\begin{array}{l}\text { Collaborator } \\
\text { heterogeneity } \\
\text { score }\end{array}$ \\
\hline Cancer prevention and control & 78 & 39 & $.82(.24)$ \\
\hline $\begin{array}{l}\text { Obesity prevention and management, including physical } \\
\text { activity, healthy eating, and obesity screening and } \\
\text { management }\end{array}$ & 75 & 40 & $.97(.05)$ \\
\hline $\begin{array}{l}\text { Tobacco use prevention and control } \\
\text { Diabetes prevention and management }\end{array}$ & 61 & 36 & $.93(.09)$ \\
\hline $\begin{array}{l}\text { Cardiovascular disease and stroke prevention } \\
\text { Multiple health promotion/non-communicable disease } \\
\text { program areas }\end{array}$ & 34 & 29 & $.82(.10)$ \\
\hline
\end{tabular}

${ }^{1}$ This $\mathrm{N}$ represents the final number of SHDs in each chronic disease program area out of a total possible maximum of 50 states. In cases of multiple respondents in a program area for a state, these were aggregated into one case.

${ }^{2}$ Score indicates probability (from a $0-1$ scale) that any two randomly selected organization types collaborating with a SHD will be in different sectors. Therefore, the closer the value is to 1 , the greater the heterogeneity a SHD will have among collaborating organization types.

\section{Results}

Response rate of the overall larger survey was $48.3 \%(n=643)$, with some additional dropout for the collaboration portion of the survey down to $(n=574)$. There were respondents from every state in the United States, but not all chronic disease area work units were represented for every state. Number of respondents for the six program areas of focus is shown in Table 1. Regarding the distribution of participation across ASTHO defined regions (i.e., New England, South, West, Mountains/Midwest, MidAtlantic/Great Lakes), there was relatively even distribution (within a range of $16.5 \%$ of total respondents in the least represented region to $23.3 \%$ in the most represented region). Most participants were program 
managers (50.7\%) or specialists in specific roles such as epidemiologist or health educator (33.0\%). Further information on participant demographics, background education and training, and evidencebased decision making capacity have been previously reported [23].

We created diagrams depicting the top 10 types of organizations SHDs collaborated with the most (regardless of sector) for each of the six SHD program areas of focus (see Figs. 1-6). Node sizes in the diagram reflect number of collaborations SHD chronic program areas have with organizations in and outside of the health sector. Thus, larger sized organization type nodes indicate a greater number of SHDs reported collaborating with a particular organization type, compared to a smaller sized node of another organization type. Blue-colored circles signify an organization type within the health sector. Green-colored circles indicate organization types from sectors outside of health. From Figs. 1-6, the obesity and tobacco program areas collaborated the most with organizations outside of the health sector, with $50 \%(5 / 10)$ and $30 \%(3 / 10)$ respectively of the organization types obesity and tobacco collaborated with coming from outside of the health sector. In cancer, cardiovascular disease, and diabetes SHD program areas, $10 \%(1 / 10)$ of SHDs most frequently collaborated with organizations came from outside of the health sector. Additional figures showing the collaboration frequency of all 26 organization types for both the health and multisector can be found in Appendix A.

These findings are supported by the collaborator heterogeneity measure shown in Table 1. If any two organization types are selected at random, the obesity (.97+/-.05) and tobacco (.93+/-.09) SHD program areas have the highest probability of the selected two organizations being from different sectors, therefore indicating these two program possessing a greater tendency of heterogeneity among their collaborators. Cancer (.82+/-.24), cardiovascular (.78+/-.21), and diabetes (.82+/-.10) program areas have the lowest probabilities of collaborators being from different sectors. In comparing heterogeneity of types of organizations SHDs collaborated with, there was a statistically significant difference in group mean heterogeneity for collaboration between program areas as determined by one-way ANOVA $(F=9.23, p$ $<.001$ ). Tukey's post hoc test suggests the obesity (mean difference +.11$)$ and tobacco (mean difference +.07 ) program areas had significantly higher group mean collaborator heterogeneity scores compared to the other chronic disease program areas.

\section{Discussion}

This study provides a look at the types of organizations SHD health promotion/chronic disease units collaborate with, the extent to which these collaborating organizations belong to the health versus other sectors, and if collaborators differ by chronic disease program area. Our results suggest that for several major chronic disease program areas, the types of organizations SHDs collaborate with remain heavily skewed towards being in the health sector. For a few select program areas (obesity and tobacco), more frequent partnerships appear to be present for sectors outside of health.

In several areas, the results of this study are congruent with findings from the most recent ASTHO national survey on cross-sector collaborations among state health agencies. Although the ASTHO survey 
did not look at collaboration by program area, they did report generally strong collaboration patterns between SHDs and organizations in the health sector [6]. For most agency types in the health sector, at least $90 \%$ of SHDs reported at least exchanging information with six of nine types of health sector agencies (hospitals, physician practices/medical groups, community health centers, health insurers, and emergency responders) [6].

The lower degree of collaborator heterogeneity among certain chronic disease areas can potentially be attributed to key factors associated with funding from government sources for activities and interventions within these program areas. For example, in the cancer program area, a larger proportion of funding may go towards screening-related activities that involve a clinical service, thus skewing collaborations in this program area more towards organizations in the health sector. Despite this, more multisector collaboration could still be encouraged, particularly in the areas of prevention and community-based living for survivors after treatment. While having many of the same mechanisms of comorbidity, diabetes and cardiovascular disease as program areas had significantly lower heterogeneity of collaborators compared to obesity. Again, although diabetes and cardiovascular disease may be more narrowly defined conditions involving specific clinical services related to diagnoses, treatment, and management compared to general obesity, these program areas could also benefit from increased multisector collaborations. Finally, it is noteworthy that multisector collaboration was not particularly high in the "multiple chronic diseases" program area. Participants that reported working across multiple program areas included epidemiologists and evaluators and other roles that may not collaborate outside the SHD. Program managers and chronic disease unit leadership teams with supervisory responsibilities across multiple program areas would not be the SHD employees to directly work with the state-wide tobacco-free coalition, for example, but is likely they would be working with Medicaid and other state-wide entities to set up collaborative initiatives.

When drawing conclusions from study results, several limitations should be considered. Importantly, our sampling method was not designed to purposively sample across all states in a complete or uniform manner. Additionally, the decision to aggregate data from multiple respondents for SHD program area into a single case may provide a clearer picture of the collaboration patterns for each SHD, but some granularity in the data may be lost. Importantly, we only asked SHD participants who they collaborate with, so the findings are unidirectional. Self-report carries the limitation of possible social desirability bias, which could inflate within-health sector reporting (e.g., CDC sometimes urges SHDs to collaborate with healthcare systems).

\section{Conclusions}

Despite limitations, our study results provide important implications for SHD multisector collaborations moving forward, particularly taking into account major issues facing the public health system today. In the context of health equity, cancer, cardiovascular disease, and diabetes are among some of the chronic diseases with the largest disparities [24, 25]. Yet in these areas, SHD collaborations remained heavily skewed to health sector organizations, which are equipped primarily to offer a narrow range of specific 
clinical services and not address multiple community and social needs; a broad array of policies; and other critical social determinants of health $[1,26]$. While services like health screenings, primary care, and hospitalization are important and readily provided by the health sector, other services such as transportation, education, housing, and income assistance linked strongly with social determinants of health are not adequately addressed by health sector organizations alone. Evidence suggests equity and community health are substantially enhanced when diverse organizational types in tandem with the health sector can work hand in hand [2]. Compared to hospitals or clinics (to which access is systemically reduced for many minorities and vulnerable populations), municipal, faith-based, social, and educational institutions and organizations are far more influential and accessible community-based settings through which chronic disease prevention efforts may be channeled [2].

Although our study primarily focuses on health department collaborations in the context of chronic disease, the return of infectious disease to the forefront of consciousness in public health due to COVID19 has highlighted more than ever the importance of strong multisector collaborations $[27,28,29]$. In managing a coordinated public health response for the pandemic, SHDs and local public health agencies should collaborate with multiple sectors, including state education agencies, school districts, restaurant associations and chains, entertainment venues, and public transportation to address issues such as social distancing protocols and reopening procedures. Thus, in addition to chronic disease, issues in the areas of health equity and infectious disease highlight more than ever the importance of multisector collaborations for public health departments.

Our findings that key chronic disease program areas still collaborate largely with health sector organizations pave several directions for future research. A direct next step is to further investigate mechanisms that facilitate collaboration of public health department work units with community-based organizations outside of the health sector. One potential approach to promoting multisector collaboration is specifically through key funding agency and stakeholder partnerships. For example, the CDC Action Communities for Health, Innovation, and EnVironmental Change (ACHIEVE) initiative brought together national organizations from different sectors (e.g. YMCA-USA, National Recreation and Parks Association, National Association of County and City Health Officials) to create a cross-agency collaborative grant funding structure for local community initiatives [1]. Effective partnerships are bidirectional and involve reciprocal investment from both parties, therefore, future research could study collaboration and the costs of collaboration from the vantage of multisector partnering organizations rather than largely from the perspective of health organizations such as public health departments. Finally, we recommend more investigation on collaboration dynamics between state-level and local-level multisector organizations, as organizations at the local level are often in the most optimal positions to promote health in neighborhoods and communities [30, 31].

\section{List Of Abbreviations}

SHD

state health department 
DHHS

Department of Health and Human Services

IOM

Institute of Medicine

ASTHO

Association of State and Territorial Health Officials

NACDD

National Association of Chronic Disease Directors

ACHIEVE

Action Communities for Health, Innovation, and EnVironmental Change

\section{Declarations}

Ethics approval and content to participate: This study was approved for human subject research by the Institutional Review Board at Washington University in St. Louis (\#201812062). Participants provided written consent to participate in the self-report survey.

Consent for publication: Not applicable.

Availability of data and materials: For related research materials, please see the Prevention Research Center in St. Louis, Washington University in St. Louis website, https://prcstl.wustl.edu/. Materials are available upon reasonable request.

Competing interests: The authors declare that they have no competing interests.

Funding: This project is funded in part by the National Cancer Institute of the National Institutes of Health (R01CA214530) for study design, data collection, and data management. Additional support for this project came from the National Cancer Institute (P50CA24431, T32CA190194) and the Centers for Disease Control and Prevention (U48DP006395) for manuscript writing, analysis, and interpretation of data. The findings and conclusions in this paper are those of the authors and do not necessarily represent the official positions of the National Institutes of Health or the Centers for Disease Control and Prevention.

Authors' contributions: Study conception and survey design: RCB, PA; Data analyses: ET; Manuscript writing: ET; Manuscript editing: PA, RCB, ET, LFS; Study management: LFS. All coauthors approved the final version of the manuscript.

Acknowledgements: N/A

\section{References}


1. Bialek, R., Beitsch, L.M., \& Moran JW. (2017). Solving population health problems through collaboration. Routledge.

2. Mazzucca, S., Arredondo, E.M., Hoelscher, D.M. et al. (2020). Expanding implementation research to prevent chronic diseases in community settings. Annual Review of Public Health, 42.

3. Association of State and Territorial Health Officials (2018). Introduction to multisector intersections and collaborations to advance health equity. https://www.astho.org/Health-

Equity/Documents/Introduction-to-Multi-Sector-Intersections-and-Collaborations-to-Advance-HealthEquity/

4. Zahner, S. J. (2005). Local public health system partnerships. Public Health Reports, 120(1), 76-83.

5. Padgett, S. M., Bekemeier, B., \& Berkowitz, B. (2004). Collaborative partnerships at the state level: Promoting systems changes in public health infrastructure. Journal of Public Health Management and Practice, 10(3), 251-257.

6. Association of State and Territorial Health Officials (2018). Promoting health in all policies: An assessment of cross-sector collaboration among state health agencies. https://www.astho.org/Prevention/Promoting-HiaP-An-Assessment-of-Cross-Sector-CollaboratingAmong-State-Health-Agencies-2018/

7. Elliott, L., McBride, T.D., \& Allen P. et al. (2014). Health care system collaboration to address chronic diseases: A nationwide snapshot from state public health practitioners. Preventing Chronic Disease, 11, E152.

8. Willis, C. D., Corrigan, C., Stockton, L., Greene, J. K., \& Riley, B. L. (2017). Exploring the unanticipated effects of multi-sectoral partnerships in chronic disease prevention. Health Policy, 121(2), 158-168.

9. Willis, C. D., Greene, J. K., Abramowicz, A., \& Riley, B. L. (2016). Strengthening the evidence and action on multi-sectoral partnerships in public health: An action research initiative. Health Promotion and Chronic Disease Prevention in Canada: Research, Policy and Practice, 36(6), 101.

10. Tabak, R. G., Parks, R. G., Allen, P. et al. (2018). Patterns and correlates of use of evidence-based interventions to control diabetes by local health departments across the USA. BMJ Open Diabetes Research and Care, 6(1).

11. Cheadle, A., Rosaschi, M., \& Burden, D. et al. (2019). A community-wide collaboration to reduce cardiovascular disease risk: The hearts of Sonoma County initiative. Preventing Chronic Disease, 16, E89.

12. Steele, C. B., Rose, J. M., Townsend, J. S., Fonseka, J., Richardson, L. C., \& Chovnick, G. (2015). Comprehensive cancer control partners' use of and attitudes about evidence-based practices. Preventing Chronic Disease, 12, E113. 
13. Bravo, R.L., Kietzman, K.G., Toy, P., Duru, O.K., \& Wallace S.P. (2019). Linking primary care and community organizations to increase colorectal cancer screening rates: The HAPPI project. Salud Publica Mex, 61(4), 427-435.

14. Phillips-Angeles, E., Song, L., Hannon, P. A., et al. (2013). Fostering partnerships and program success. Cancer, 119, 2884-2893.

15. Stoto, M. A., Davis, M. V., \& Atkins, A. (2019). Beyond CHNAS: Performance measurement for community health improvement. eGEMs, $7(1), 45$.

16. Berkowitz, B. (2000). Collaboration for health improvement: Models for state, community, and academic partnerships. Journal of Public Health Management and Practice, 6(1), 67-72.

17. Furtado, K.S., Brownson, C., Fershteyn, Z. et al. (2018). Health departments with a commitment to health equity: A more skilled workforce and higher-quality collaborations. Health Affairs, 7(1), 38-46.

18. Garzón-Orjuela, N., Samacá-Samacá, D. F., Angulo, S. C. L., Abdala, C. V. M., Reveiz, L., \& EslavaSchmalbach, J. (2020). An overview of reviews on strategies to reduce health inequalities. International journal for equity in health, 19(1), 1-11.

19. Padek, M., Allen, P., Erwin, P. C., et al. (2018). Toward optimal implementation of cancer prevention and control programs in public health: A study protocol on mis-implementation. Implementation Science, 13(1), 1-13.

20. Agresti, A., \& Agresti, B. F. (1978). Statistical analysis of qualitative variation. Sociological methodology, 9, 204-237.

21. Perry, B. L., Pescosolido, B. A., \& Borgatti, S. P. (2018). Egocentric network analysis: Foundations, methods, and models(Vol. 44). Cambridge University Press.

22. Borgatti, S.P. (2006). E-Network Software for Ego-Network Analysis. Analytic Technologies: Lexington, $\mathrm{KY}$.

23. Padek, M., Mazzucca, S., Allen, P. et al. (2021). Patterns and correlates of mis-implementation in state chronic disease public health practice in the United States. BMC Public Health.

24. Minas, T. Z., Kiely, M., Ajao, A., \& Ambs, S. (2020). An overview of cancer health disparities: New approaches and insights and why they matter. Carcinogenesis. bgaa121.

25. Caspard, H., Jabbour, S., Hammar, N., Fenici, P., Sheehan, J. J., \& Kosiborod, M. (2018). Recent trends in the prevalence of type 2 diabetes and the association with abdominal obesity lead to growing health disparities in the USA: An analysis of the NHANES surveys from 1999 to 2014. Diabetes, Obesity and Metabolism, 20(3), 667-671. 
26. Provan, K. G., Veazie, M. A., Teufel-Shone, N. I., \& Huddleston, C. (2004). Network analysis as a tool for assessing and building community capacity for provision of chronic disease services. Health Promotion Practice, 5(2), 174-181.

27. Landers, G. M., Minyard, K. J., Lanford, D., \& Heishman, H. (2020). A theory of change for aligning health care, public health, and social services in the time of COVID-19. American Journal of Public Health, 110, S178-S180.

28. Park, J. \& Chung, E. (2020). Learning from past pandemic governance: Early response and publicprivate partnerships in testing of COVID-19 in South Korea. World Development, 137, 105198.

29. Baxter, D., \& Casady, C. B. (2020). Proactive and strategic healthcare public-private partnerships (PPPs) in the coronavirus (COVID-19) epoch. Sustainability, 12(12), 5097.

30. Roussos, S. T. \& Fawcett, S. B. (2000). A review of collaborative partnerships as a strategy for improving community health. Annual Review of Public Health, 21(1), 369-402.

31. Baker, E. L., Melton, R. J., Stange, P.V.et al. (1994). Health reform and the health of the public: Forging community health partnerships. JAMA, 272(16), 1276-1282.

\section{Figures}




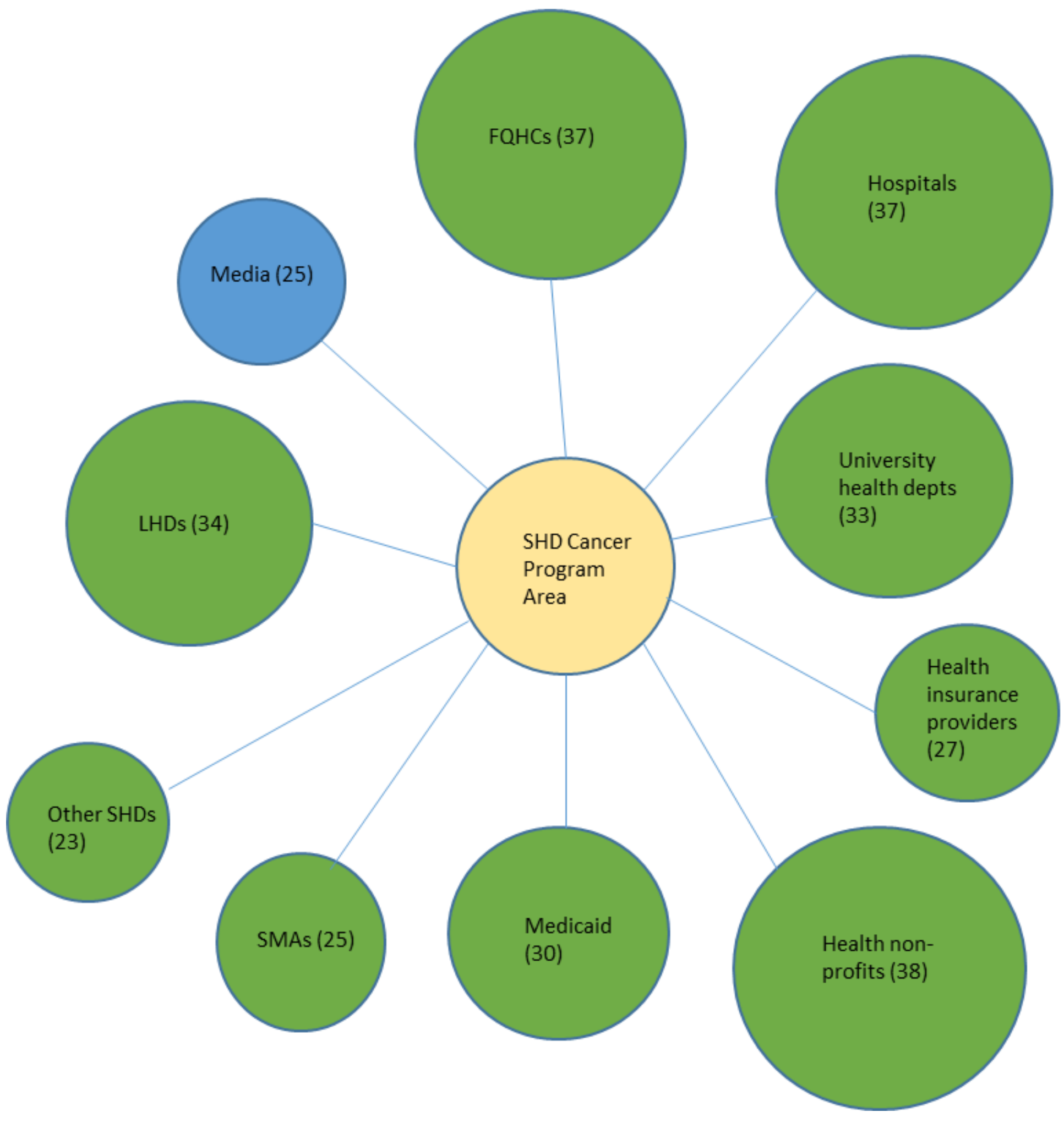

Figure 1

Top 10 SHD collaborators in the cancer program area 


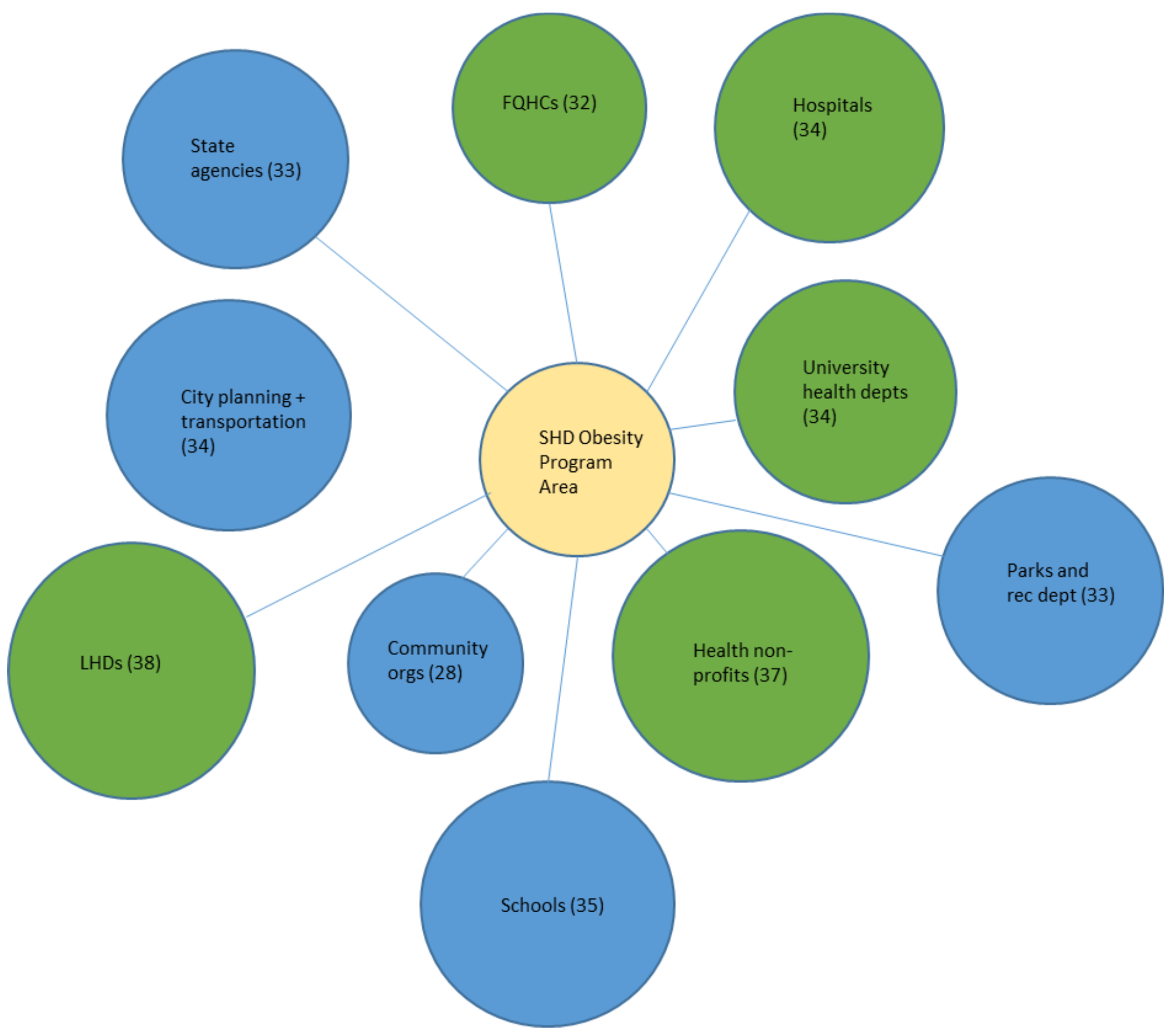

Figure 2

Top 10 SHD collaborators in the obesity program area 


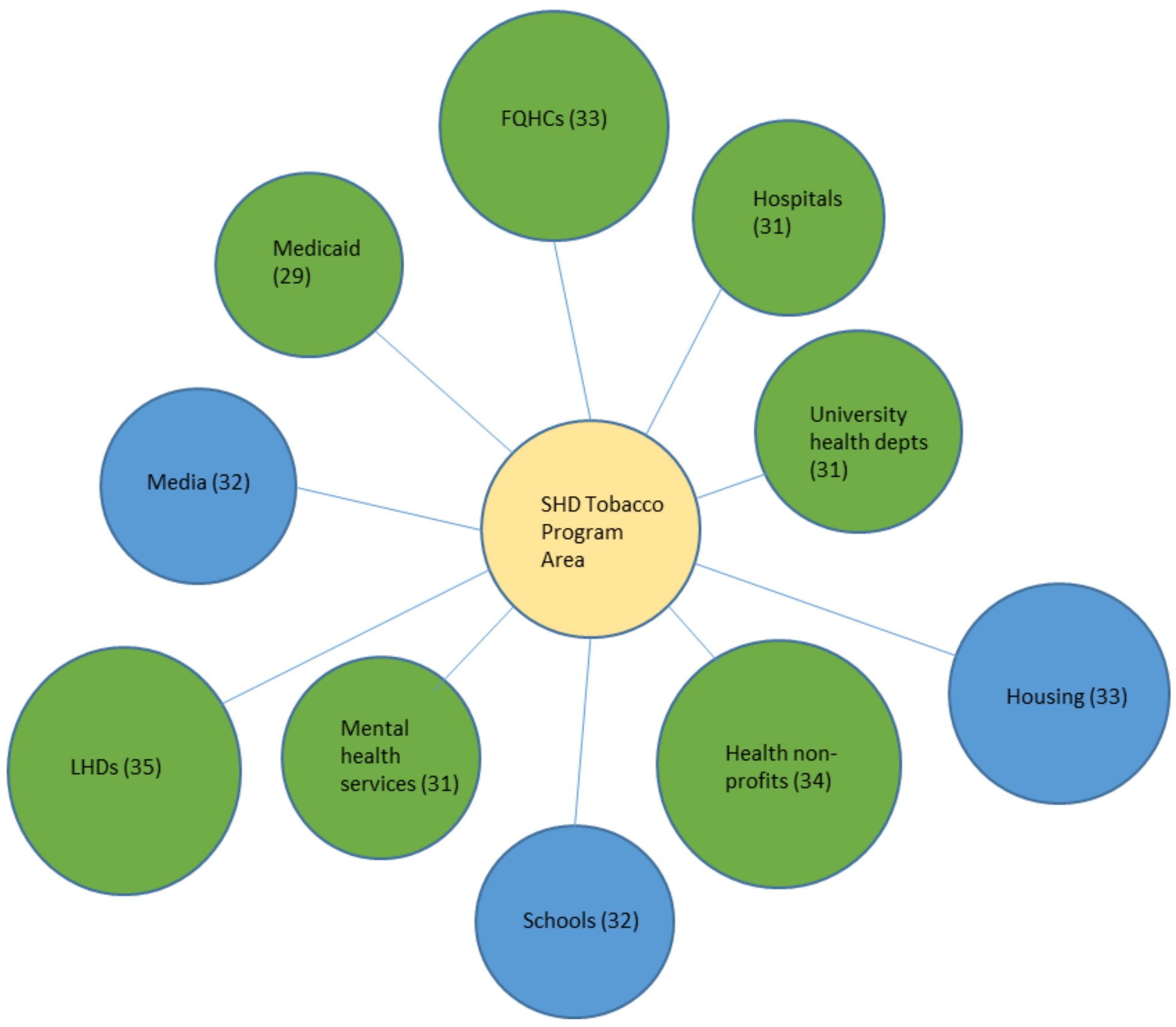

Figure 3

Top 10 SHD collaborators in the tobacco program area 


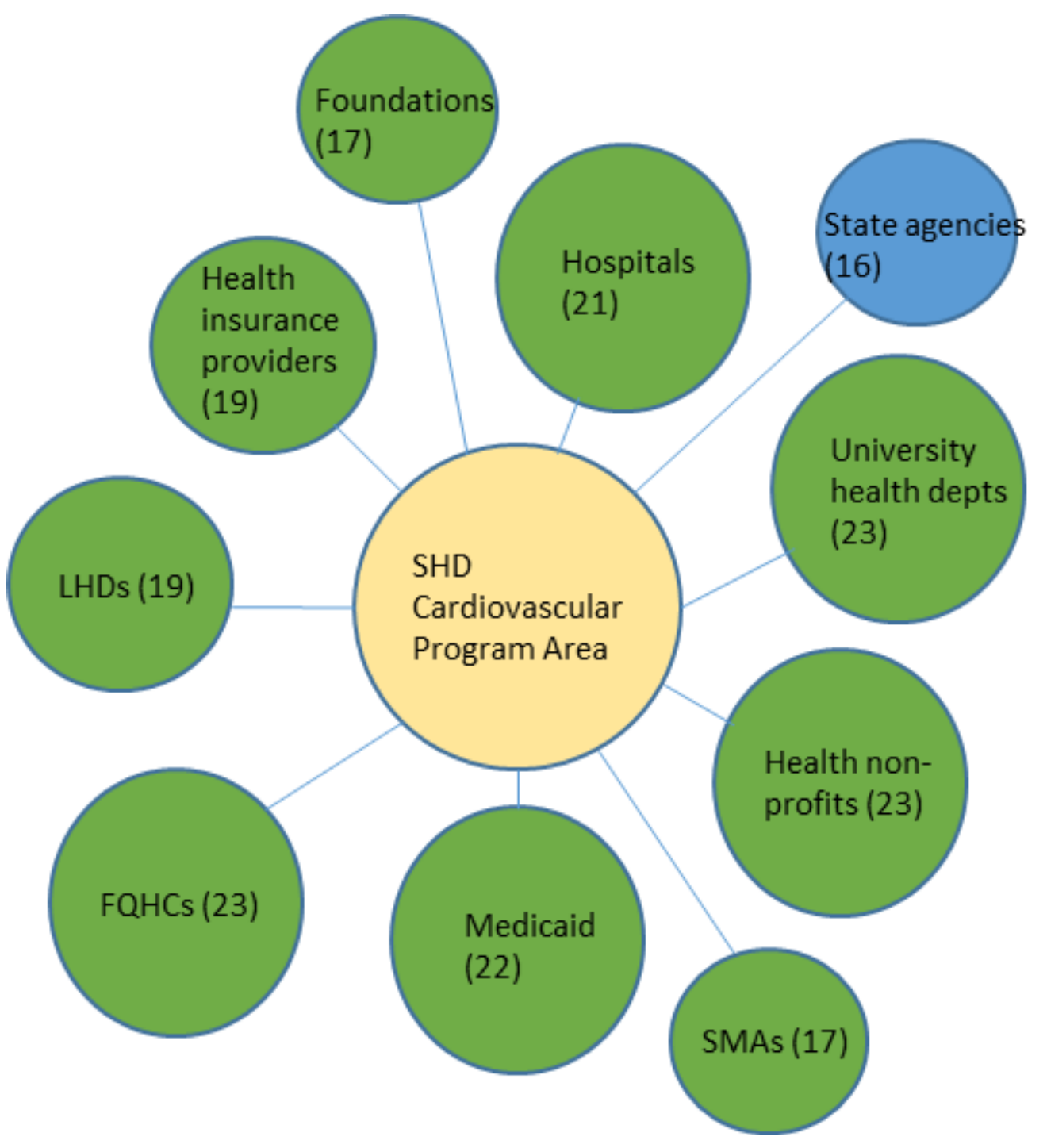

Figure 4

Top 10 SHD collaborators in the cardiovascular program area 


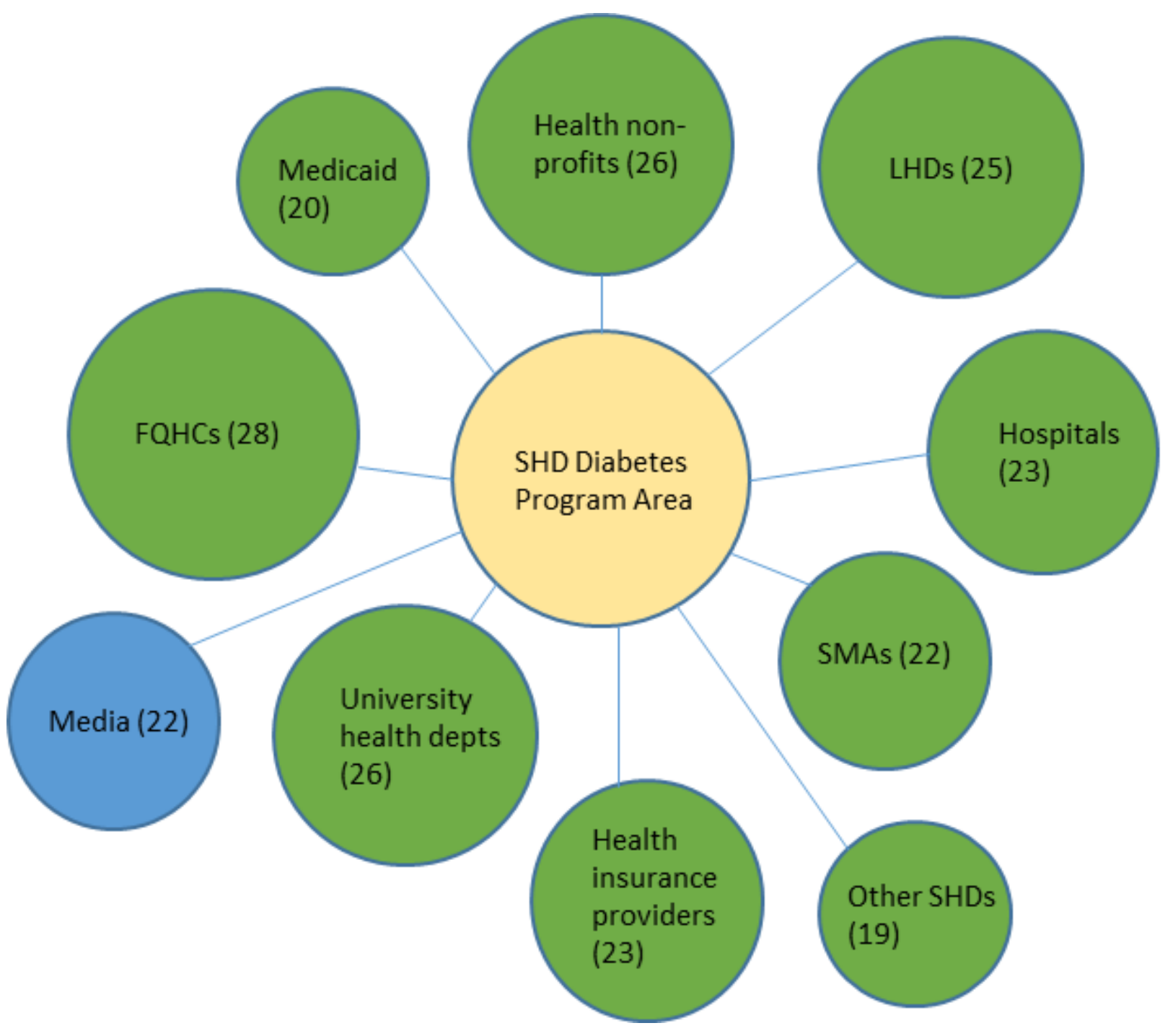

Figure 5

Top 10 SHD collaborators in the diabetes program area 


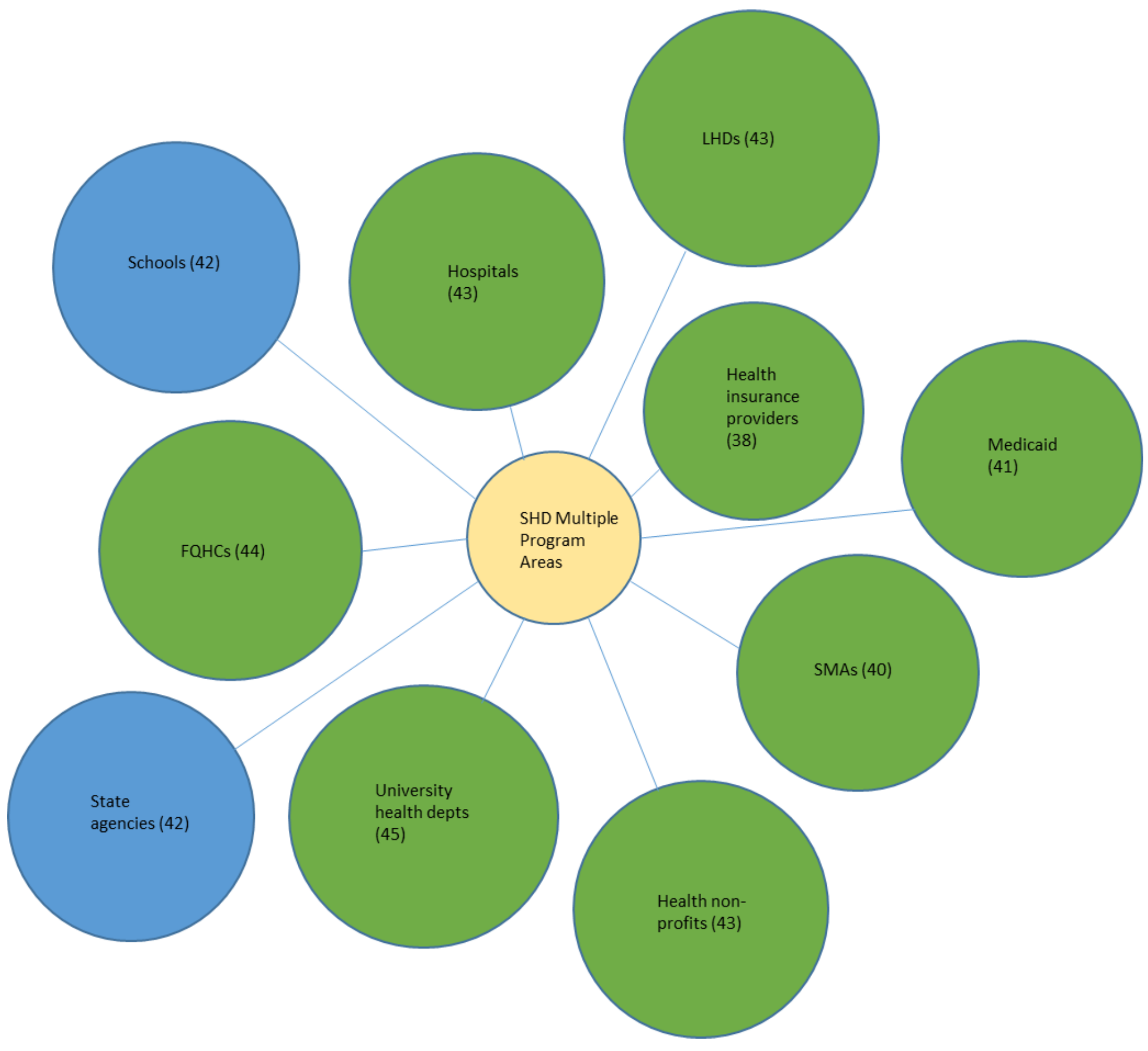

Figure 6

Top 10 SHD collaborators in the "working in multiple chronic disease program areas"

\section{Supplementary Files}

This is a list of supplementary files associated with this preprint. Click to download.

- AppendixA.docx 